\title{
Nuclear Mass Measurements Map the Structure of Atomic Nuclei and Accreting Neutron Stars
}

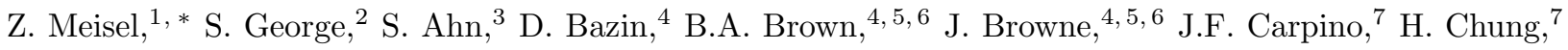 \\ R.H. Cyburt, ${ }^{4,5}$ A. Estradé, ${ }^{8}$ M. Famiano, ${ }^{7}$ A. Gade, ${ }^{4,6}$ C. Langer, ${ }^{9}$ M. Matoš, ${ }^{10}$ W. Mittig, ${ }^{4,6}$ F. Montes,${ }^{4,5}$ \\ D.J. Morrissey, ${ }^{4,11}$ J. Pereira, ${ }^{4,5}$ H. Schatz,${ }^{4,5,6}$ J. Schatz, ${ }^{4}$ M. Scott, ${ }^{4,6}$ D. Shapira, ${ }^{12}$ K. Smith, ${ }^{13}$ J. Stevens,${ }^{4,5,6}$ \\ W. Tan, ${ }^{14}$ O. Tarasov, ${ }^{4}$ S. Towers,${ }^{7}$ K. Wimmer, ${ }^{15}$ J.R. Winkelbauer, ${ }^{13}$ J. Yurkon, ${ }^{4}$ and R.G.T. Zegers ${ }^{4,5,6}$ \\ ${ }^{1}$ Institute of Nuclear $\& 3$ Particle Physics, Department of Physics \& Astronomy, Ohio University, Athens, Ohio 45701, USA \\ ${ }^{2}$ Universität Greifswald, Institut für Physik, Greifswald 17487, Germany \\ ${ }^{3}$ Cyclotron Institute, Texas A $6 M$ University, College Station, Texas 77843, USA \\ ${ }^{4}$ National Superconducting Cyclotron Laboratory, Michigan State University, East Lansing, Michigan 48824, USA \\ ${ }^{5}$ Joint Institute for Nuclear Astrophysics - Center for the Evolution of the Elements, \\ Michigan State University, East Lansing, Michigan 48824, USA \\ ${ }^{6}$ Department of Physics and Astronomy, Michigan State University, East Lansing, Michigan 48824, USA \\ ${ }^{7}$ Department of Physics, Western Michigan University, Kalamazoo, Michigan 49008, USA \\ ${ }^{8}$ Department of Physics, Central Michigan University, Mount Pleasant, Michigan 48859, USA \\ ${ }^{9}$ Department of Energy Technology, University of Applied Science Aachen, Campus Jülich, 52428 Jülich, Germany \\ ${ }^{10}$ Physics Section, International Atomic Energy Agency, Vienna 1400, Austria \\ ${ }^{11}$ Department of Chemistry, Michigan State University, East Lansing, Michigan 48824, USA \\ ${ }^{12}$ Oak Ridge National Laboratory, Oak Ridge, Tennessee 37831, USA \\ ${ }^{13}$ Los Alamos National Laboratory, Los Alamos, New Mexico 87545, USA \\ ${ }^{14}$ Department of Physics, University of Notre Dame, Notre Dame, Indiana 46556, USA \\ ${ }^{15}$ Department of Physics, University of Tokyo, Hongo 7-3-1, Bunkyo-ku, Tokyo 113-0033, Japan
}

(Dated: April 30, 2020)

\begin{abstract}
We present mass excesses (ME) of neutron-rich isotopes of Ar through Fe, obtained via TOF$B \rho$ mass spectrometry at the National Superconducting Cyclotron Laboratory. Our new results have significantly reduced systematic uncertainties relative to a prior analysis, enabling the first determination of $\mathrm{ME}$ for ${ }^{58,59} \mathrm{Ti},{ }^{62} \mathrm{~V},{ }^{65} \mathrm{Cr},{ }^{67,68} \mathrm{Mn}$, and ${ }^{69,70} \mathrm{Fe}$. Our results show the $N=34$ subshell weaken at $\mathrm{Sc}$ and vanish at $\mathrm{Ti}$, along with the absence of an $N=40$ subshell at Mn. This leads to a cooler accreted neutron star crust, highlighting the connection between the structure of nuclei and neutron stars.
\end{abstract}

The rest mass $m$ is a basic property of an atomic nucleus, essential for calculating astrophysical processes such as X-ray burst light curves and $r$-process nucleosynthesis, and key to mapping the evolution of nuclear structure across the nuclear landscape $1-3$. While nuclear masses nearly follow the whole-number rule, $m \approx A m_{u}$, where $A$ is the mass number and $m_{u}=931.49 \mathrm{MeV}$ is the atomic mass unit, the $\lesssim 1 \%$ deviation from this relationship due to nuclear binding is notoriously difficult to predict. State-of-the-art mass models [e.g. 44] often disagree in their predictions of the atomic mass excess, $\operatorname{ME}(Z, A)=m-(Z+N) m_{u}$ where $Z$ is the proton number and $N$ is the neutron number, by more than one $\mathrm{MeV}$. Similar discrepancies are present when comparing predictions to experimentally measured masses. As such, experiments mapping the evolution of the nuclear mass surface across the nuclear landscape are essential.

For neutron-rich nuclides, mass measurements have revealed the emergence and disappearance of the magic numbers that indicate enhanced nuclear binding [e.g 8 15. For instance, $N=34$ semi-magicity emerges for neutron-rich calcium isotopes [14, whereas there are signatures that the $N=40$ harmonic oscillator subshell gap disappears for neutron-rich manganese [16]. While the evolution of these subshells were mapped by spectroscopy experiments that often long-preceded the corresponding mass measurements [e.g. 17 24], nuclear masses provided the first model-independent confirmation of this spectroscopic evidence via fundamental ground state properties.

These evolutions in nuclear structure are closely linked to the thermal structure of accreting neutron stars. Nuclei produced by surface burning processes are buried by subsequent accretion, resulting in a number of nuclear reactions in the neutron star crust that drive it from thermal equilibrium 25]. Electron-capture (EC) reactions near closed shells result in relatively large ECheating due to the large change in the EC $Q$-value $Q_{\mathrm{EC}}=\operatorname{ME}(Z, A)-\operatorname{ME}(Z-1, A)$ [26]. $\mathrm{EC}$ in regions of deformation between closed shells occur on isobars with a small odd-even staggering in $Q_{\mathrm{EC}}$ as well as lowlying excited states, which results in the EC- $\beta^{-}$-decay cycling process known as urca cooling [27. Therefore, whether EC heating or cooling occurs, and the strength of the heat source or heat sink, strongly depend on nuclear masses 28,30$]$.

To simultaneously map the evolution of the $N=34$ and $N=40$ subshells and constrain the thermal structure of accreting neutron stars, we performed mass measurements of neutron-rich isotopes of $\mathrm{Ar}$ through $\mathrm{Fe}$ $(Z=18-26)$. First results from these measurements 
have been reported in Refs. [10, 29, 30. The present work is a re-evaluation of the original data, incorporating recently published high-precision Penning trap mass data [12, 13] as additional calibration nuclides, which greatly reduces the systematic uncertainty present in our results and greatly expands the number of nuclides for which masses are obtained. Our results extend the known nuclear mass surface, provide model-independent confirmation of the emergence of $N=34$ and disappearance of $N=40$ semimagicity, and significantly update predictions for urca cooling in accreted neutron star crusts.

Magnetic-rigidity corrected time-of-flight (TOF- $B \rho$ ) mass measurements were performed at the National Superconducting Cyclotron Laboratory. The measurement technique and measurements are described in detail in Refs. [10, 29,32] and are only briefly summarized here. A $140 \mathrm{MeV} /$ nucleon beam of ${ }^{82} \mathrm{Se}$ accelerated by the coupled cyclotrons impinged on a Be target and the resulting fully-stripped (charge $q=Z$ ) fragments were transmitted through the A1900 fragment separator 33, momentum-analyzed at the target location of the S800 spectrograph [34, and stopped in the focal plane of the S800 35. Particle identification was performed eventby-event using the TOF- $\Delta E$ method, where TOF was provided by fast-timing scintillators separated by a flight path $L_{\text {path }}=60.6 \mathrm{~m}$ and energy loss $\Delta E$ determined using an ionization chamber. A relative measurement of $B \rho$, which is the momentum over $q$, was obtained via a position measurement using a microchannel plate detector located at the dispersive focus of the S800 [36, 37.

Nominally, $m=\left(\mathrm{TOF} / L_{\text {path }}\right)(q B \rho / \gamma)$, where $\gamma$ is the Lorentz factor. However, determining $L_{\text {path }}$ and $B \rho$ to sufficient precision is not practicable. Instead, an empirical relationship between $m / q$ and TOF is determined from a fit to nuclides of known $m$ which are simultaneously measured alongside nuclides of interest. This work improves on prior results [10, 29, 30] by including seven additional calibration nuclides, bringing the total to 27. High-precision $\mathrm{ME}$ for ${ }^{59-63} \mathrm{Cr}\left[13\right.$ and ${ }^{54,55} \mathrm{Ti}[12$ ] substantially improve constraints on the $m / q$ (TOF) relationship, whose ambiguity previously provided one of the dominant contributions to our ME uncertainties [30].

Mass fits were performed as described in Ref. 30]. Several fit functions were explored, of the form

$$
\frac{m}{q}(\tau)=a_{0}+a_{1} \tau+a_{2} z+a_{3} \tau^{2}+a_{4} z^{2}+a_{5} z \tau+f(z, \tau),
$$

where $\tau=\mathrm{TOF}-\langle\mathrm{TOF}\rangle, z=Z-\langle Z\rangle$, and $f(z, \tau)$ is a function of higher-order in $z$ and/or $\tau$. The addition of the new $\mathrm{Ti}$ and $\mathrm{Cr}$ reference nuclides resolved the previously existing ambiguity in the $Z$-dependence, while the $\mathrm{Cr}$ masses additionally clarified that a higherorder TOF component was needed to adequately minimize fit residuals, which are shown for the best-fit in Fig. 1. The best-fit function has $f(z, \tau)=a_{6} z^{3}+a_{7} \tau^{4}$, whereas a fit of slightly lower quality was obtained with $f(z, \tau)=a_{6} z^{3}+a_{7} \tau^{3}$. This set of acceptable functions was determined by the following criterion. (1) The fit residuals must lack systematic trends. (2) The fit residuals must be robust to the arbitrary removal of reference nuclides. (3) The difference between $\chi^{2}$ for a fit function and the best-fit $\Delta \chi_{i}^{2}=\chi_{i}^{2}-\chi_{\min }^{2}$ must be within three standard deviations of the best-fit $\left(\Delta \chi_{i}^{2} \lesssim 37\right)$, which is a valid metric based on the Gaussian distribution of the fit parameters after repeated fits varying ME for reference nuclides in a Monte Carlo procedure [30, 38].

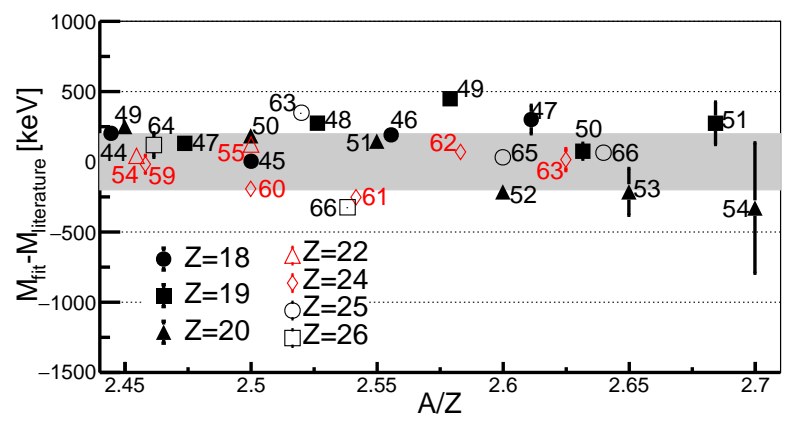

FIG. 1. Residuals of the $m / q$ (TOF) fit to calibration nuclides, where the isotope of an element is indicated by the number next to the data point. Red data are new calibration nuclides in this re-evaluation. The gray band represents the average systematic mass uncertainty from the $\chi^{2}$ normalization.

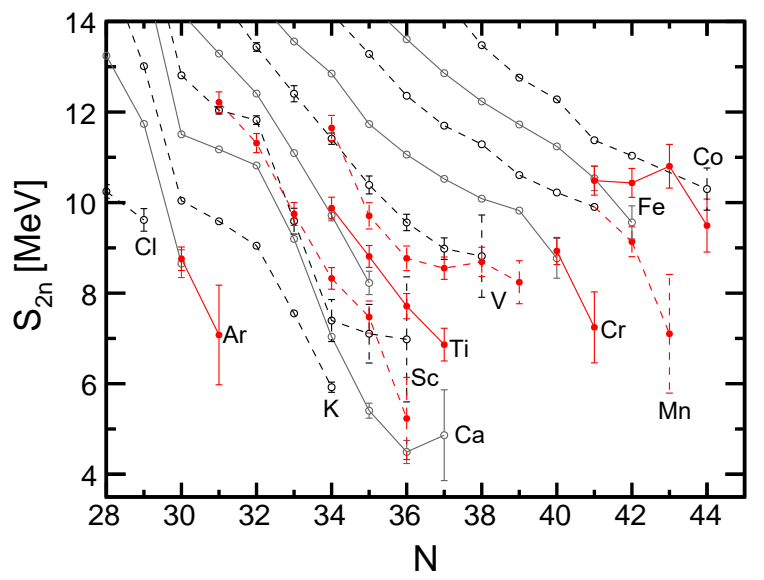

FIG. 2. $S_{2 n}$ for isotopes of $\mathrm{Cl}$ (left-most trend line) through Co (right-most), where the black and gray open circles correspond to values using the 2016 AME [39] or higher-precision ME from the subsequent literature [12, 13, 40, 41], dashed lines are odd- $Z$, and solid lines are even- $Z$. Red filled circles are from this work.

The smaller number and closer similarity of the set of acceptable functions substantially reduce the fitfunction uncertainty relative to the previous evalua- 
tion [30. Additional uncertainty contributions come from the $9.1 \mathrm{keV} / q$ systematic uncertainty added to all nuclides to normalize the reduced $\chi^{2}$ to one for the bestfit and the uncertainty in $a_{i}$ due to TOF uncertainties in the reference nuclides. See Ref. 30 for details.

Our resultant ME are reported in Tabs. I and II with comparisons to literature values and results from our previous evaluation, where Fig. 2 shows the two neutron separation energy $S_{2 n}(Z, A)=2 \mathrm{ME}(0,1)+\mathrm{ME}(Z, A-2)-$ $\operatorname{ME}(Z, A)$. All but one of our updated ME are within 1 standard deviation $\sigma$ of our previous ME and all are within $2 \sigma$, while the majority of uncertainties have been reduced by a factor of two. We report ME for ${ }^{58,59} \mathrm{Ti}$, ${ }^{62} \mathrm{~V},{ }^{65} \mathrm{Cr},{ }^{67,68} \mathrm{Mn}$, and ${ }^{69,70} \mathrm{Fe}$ for the first time.

The new trend in $S_{2 n}$ for $\mathrm{V}$ is largely due to ${ }^{58,59} \mathrm{~V}$, which are much less bound in our work compared to the 2016 AME evaluated result, but in agreement with the privately communicated results Ref. 39] refers to as 1998Ba. A that were included in that evaluation. The abnormal behavior for Fe in $S_{2 n}$ shown in Fig. 2 is difficult to understand in terms of nuclear structure effects, since a new single-particle orbital is not expected to be filled. Additionally, anomalous behavior of $m / q$ (TOF) is an unlikely explanation, since the function is smooth in that region and a similar feature is not seen for nearby $Z .{ }^{67} \mathrm{Fe}$ is known to have an isomeric state at $387 \mathrm{keV}$ [42, which, along with measurement uncertainties, may explain the $\sim 1 \mathrm{MeV}$ deviation in $S_{2 n}$ from a smooth trend. We suspect ${ }^{69} \mathrm{Fe}$ may also have a long-lived isomer based on the presence of such states in odd- $A$ isotopes of Fe due to intrusion from the $\nu g_{9 / 2}$ orbital [43]. The kink at $N=39$ for Cr agrees with the trend calculated using the LNPS' Hamiltonian [13, 30, 44, but the absolute $S_{2 n}$ are discrepant.

ME for $N=36$ isotopes of $\mathrm{Sc}, \mathrm{Ti}$, and $\mathrm{V}$ can be used to deduce the evolution of $N=34$ in this region. Ref. [14] demonstrated the presence of $N=34$ semi-magicity for $\mathrm{Ca}$, whereas spectroscopy has indicated this subshell closure is absent for Ti and likely weakened for Sc, with the caveat that $E\left(2_{1}^{+}\right)$energies can provide ambiguous constraints on shell gaps [45]. Our data reveal a continuous slope in $S_{2 n}$ for Ti following $N=34$, while Sc trends slightly more negative beyond this point. This indicates that a weak $N=34$ subshell gap is present at Sc.

Further insight is provided by the trend in $D_{n}(Z, A)=$ $(-1)^{N+1}\left[S_{n}(Z, A+1)-S_{n}(Z, A)\right]$, which is related to the empirical pairing gap [46]. $D_{n}$ is proportional to the number of angular momentum projection states $(2 j+1)$ participating in pairing, providing a signature of gaps in single particle levels. Fig. 3(a) shows the trend in $D_{n}$ near $N=34$ for isotopes of Ca through V. While the dip after $N=34$ for the Sc isotopes might initially appear to be the signature of a significant shell gap, shell model calculations suggest this is not the case. Calculations using the GX1A Hamiltonian [47, whose results are shown in Fig. 3(b) to be in qualitative agreement
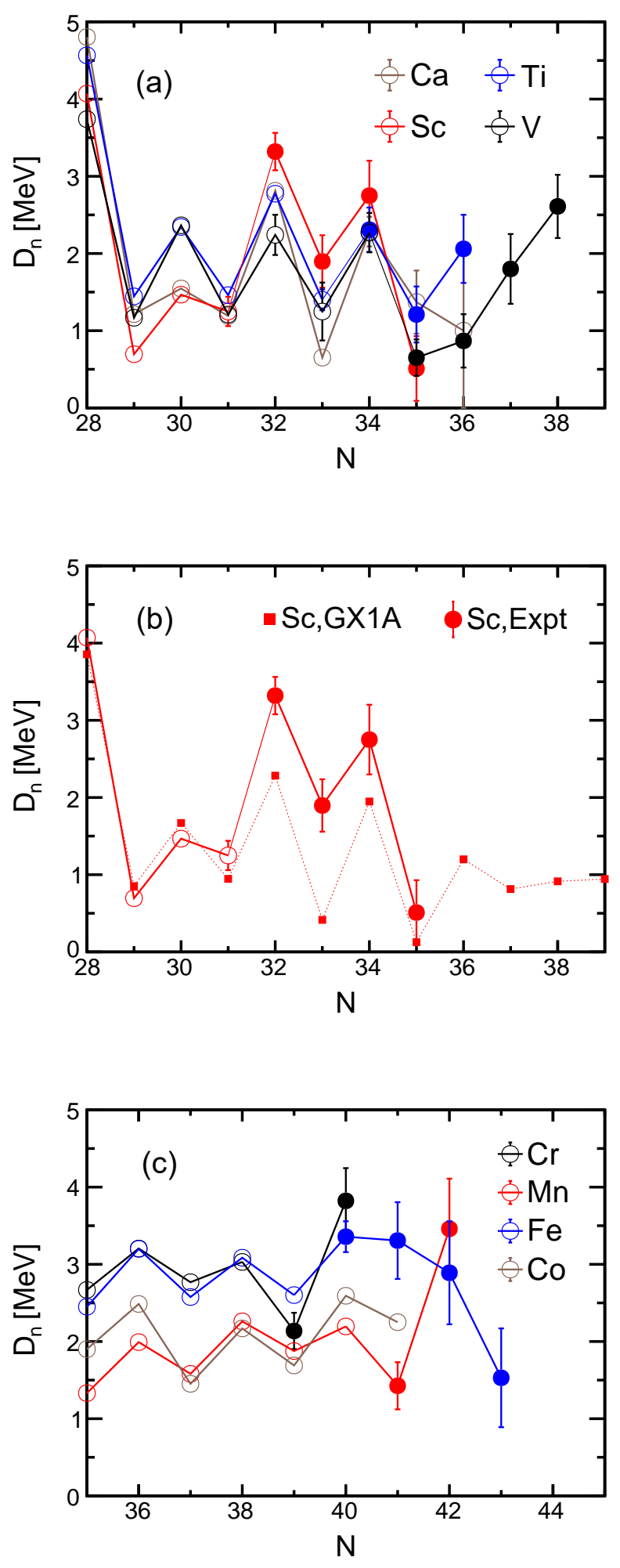

FIG. 3. $D_{n}$ using our ME (filled circles) and ME from the literature (open circles) (a) near $N=34$ and (c) near $N=$ 40. A comparison to shell model calculations with the GX1A Hamiltonian (filled-squares) is shown for Sc in (b). 
TABLE I. Atomic mass excesses (in keV) of nuclides determined in this work compared to the previous evaluation 10, 29, 30, the 2016 Atomic Mass Evaluation (AME) [39, and literature published after the 2016 AME. A * near the AME value indicates this is an extrapolation and not directly based on experimental data. The $I$ following an isotope indicates a known or suspected long-lived (> $100 \mathrm{~ns}$ ) isomeric component. For instance, for ${ }^{67} \mathrm{Fe}$ the known isomer at $387 \mathrm{keV}$ excitation energy is responsible for the additional asymmetric error bar, while for ${ }^{69} \mathrm{Fe}$ our results should be interpreted as an upper bound.

\begin{tabular}{lcccc}
\hline \hline Isotope & This Work & Previous Evaluation & AME 2016 & Literature \\
\hline${ }^{48} \mathrm{Ar}$ & $-22390(260)$ & $-22280(310)$ & $-22280(310)$ & $-22330(120)[14]$ \\
${ }^{49} \mathrm{Ar}$ & $-16300(1100)$ & $-17820(1100)$ & $-17190^{*}\left(400^{*}\right)$ & $\ldots$ \\
${ }^{52} \mathrm{Sc}$ & $-40620(230)$ & $-40300(520)$ & $-40443(82)$ & $-40525(65)[15]$ \\
${ }^{53} \mathrm{Sc}$ & $-38400(210)$ & $-38170(570)$ & $-38906(94)$ & $-38910(80)[15]$ \\
${ }^{54} \mathrm{Sc}$ & $-34050(240)$ & $-33750(630)$ & $-33890(273)$ & $-34485(360)[15]$ \\
${ }^{55} \mathrm{Sc}$ & $-31090(220)$ & $-30520(580)$ & $-30159(454)$ & $\ldots$ \\
${ }^{56} \mathrm{ScI}$ & $-25380(260)\left({ }_{-540}^{+0}\right)$ & $-24850(590)\left({ }_{-540}^{+0}\right)$ & $-24852(587)$ & $\ldots$ \\
${ }^{57} \mathrm{Sc}$ & $-20180(880)$ & $-21010(1320)$ & $-20996(1304)$ & $\ldots$ \\
${ }^{56} \mathrm{Ti}$ & $-39480(240)$ & $\ldots$ & $-39320(121)$ & $-39810(190)[15$ \\
${ }^{64} \mathrm{Cr}$ & $-33640(300)$ & $-33480(440)$ & $-33480(440)$ & $\ldots$ \\
\hline \hline
\end{tabular}

TABLE II. Table I continued, for cases without a Previous Evaluation or Literature value.

\begin{tabular}{lcc}
\hline \hline Isotope & This Work & AME 2016 \\
\hline${ }^{57} \mathrm{Ti}$ & $-34500(240)$ & $-33916(256)$ \\
${ }^{58} \mathrm{Ti}$ & $-30890(250)$ & $-31110^{*}\left(200^{*}\right)$ \\
${ }^{59} \mathrm{Ti}$ & $-25220(270)$ & $-25510^{*}\left(200^{*}\right)$ \\
${ }^{57} \mathrm{~V}$ & $-44650(260)$ & $-44413(80)$ \\
${ }^{58} \mathrm{~V}$ & $-39720(230)$ & $-40402(89)$ \\
${ }^{59} \mathrm{~V}$ & $-37040(260)$ & $-37832(162)$ \\
${ }^{60} \mathrm{VI}$ & $-32810(230)\left(_{-202}^{+0}\right)$ & $-33242(220)$ \\
${ }^{61} \mathrm{~V}$ & $-30380(280)$ & $-30506(894)$ \\
${ }^{62} \mathrm{~V}$ & $-25340(420)$ & $-25476^{*}\left(298^{*}\right)$ \\
${ }^{65} \mathrm{Cr}$ & $-27280(780)$ & $-28220^{*}\left(300^{*}\right)$ \\
${ }^{67} \mathrm{Mn}$ & $-33960(330)$ & $-33460^{*}\left(300^{*}\right)$ \\
${ }^{68} \mathrm{Mn}$ & $-27710(1310)$ & $-28380^{*}\left(400^{*}\right)$ \\
${ }^{67} \mathrm{Fe} \mathrm{C}$ & $-45560(320)\left({ }_{-387}^{+0}\right)$ & $-45610(270)$ \\
${ }^{68} \mathrm{Fe}$ & $-44360(320)$ & $-43487(365)$ \\
${ }^{69} \mathrm{Fe} \mathrm{I}$ & $-40270(400)\left({ }_{-?}^{+0}\right)$ & $-39030^{*}\left(400^{*}\right)$ \\
${ }^{70} \mathrm{Fe}$ & $-37710(490)$ & $-36510^{*}\left(400^{*}\right)$ \\
\hline \hline
\end{tabular}

with experiment, indicate that the particularly low $D_{n}$ for ${ }^{56} \mathrm{Sc}$ is due to the large splitting of levels created by the residual interaction between the $\pi f_{7 / 2}$ and $\nu f_{5 / 2}$ orbitals. In particular, the minimum in $D_{n}$ for Sc is due to the low-lying $J=1$ level created by this interaction. $S_{2 n}$ is not sensitive to this effect as it reflects the energetics of neutron shells and not the strength of proton-neutron pairing [3, 16]. These results confirm previous indications from spectroscopy of weak $N=34$ magicity for Sc, while removing the ambiguity inherent to spectroscopic interpretations of shell structure [18, 20, 45].

Fig. 2 demonstrates a continuous slope in $S_{2 n}$ through
$N=40$ for Mn, strengthening the conclusions of Ref. [16] that the $N=40$ subshell is absent for this element. This is bolstered by the trend in $D_{n}$ shown in Fig. 3(c). Our data are ambiguous regarding the $N=40$ subshell at $\mathrm{Cr}$, where the mass of ${ }^{66} \mathrm{Cr}$ is needed to confirm prior spectroscopic and coulomb-excitation evidence [e.g. 23, 48, 49.

The evolution in nuclear structure presented here is directly linked to the thermal structure of accreting neutron stars. The neutrino luminosity from urca cooling $L_{\nu} \propto X(A)(f t)^{-1}\left|Q_{\mathrm{EC}}\right|^{5}$, where $f t$ is the comparative half-life and $X(A)$ is the mass fraction, and is therefore very sensitive to changes in ME [50, 51. This process operates in the accreted neutron star crust with consequential $L_{\nu}$ for odd- $A$ nuclides with $X(A) \gtrsim 0.5 \%, f t \lesssim 5$, and $8 \lesssim\left|Q_{\mathrm{EC}}\right| \lesssim 15 \mathrm{MeV}$ [27, 52, where the upper limit on $Q_{\mathrm{EC}}$ is due to competing EC reaction channels 53 . Two EC parents predicted to produce some of the largest $L_{\nu}$, with potentially observable consequences [51, 52], are ${ }^{55} \mathrm{Sc}$ and ${ }^{65} \mathrm{Mn}$. For ${ }^{65} \mathrm{Mn}$, $f t$ will be uncertain by orders of magnitude until measurements are enabled by nextgeneration rare isotope beam facilities, but estimates from QRPA calculations 27] and using the Moszkowski nomographs 54 result in $\log (f t) \approx 5$, allowing significant $L_{\nu} \cdot{ }^{55} \mathrm{Sc}$ by contrast has more consistent predictions, with $\log (f t) \approx 5$ using QRPA methods [27, the Moszkowski nomographs, and empirical systematics [55], currently making it the highest predicted $L_{\nu}$ urca cooling layer 52, 56]. Furthermore, a measurement of $\mathrm{ft}$ for this transition has recently taken place [57]. For both $A=55$ and $65, X(A)>0.5 \%$ and are remarkably consistent for a wide variety of assumptions for nuclear burning on the accreting neutron star surface [58]. Therefore $Q_{\mathrm{EC}}$ are the final piece of the nuclear physics puzzle for these urca coolers.

Using the newly determined $\mathrm{ME}\left({ }^{55} \mathrm{Sc}\right),\left|Q_{\mathrm{EC}}\left({ }^{55} \mathrm{Sc}\right)\right|=$ 
$12.44(0.27) \mathrm{MeV}$, to be compared to the prior [14, 39] value $11.51(0.48) \mathrm{MeV}$. Our reported $\mathrm{ME}\left({ }^{65} \mathrm{Cr}\right)$ results in $\left|Q_{\mathrm{EC}}\left({ }^{65} \mathrm{Mn}\right)\right|=13.69(0.78) \mathrm{MeV}$, to be compared to the prior [16, 39] value $12.75(0.30) \mathrm{MeV}$ (though the latter uncertainty relies on the AME extrapolation, which assumes an essentially featureless nuclear mass surface and may be underestimated). These results increase $L_{\nu}$ by $50 \%$ for $\mathrm{EC}$ on ${ }^{55} \mathrm{Sc}$ with half the prior uncertainty and provide the first experimental determination of $L_{\nu}$ for EC on ${ }^{65} \mathrm{Mn}$. While in agreement with previous predictions, our central value for $Q_{\mathrm{EC}}\left({ }^{65} \mathrm{Mn}\right)$ leads to $40 \%$ larger $L_{\nu}$. Therefore the accreted neutron star crust is cooler than previously thought, with an improved precision on the description of the neutron star thermal structure.

In conclusion, this work highlights the intriguing connection between evolution in nuclear structure and the thermal structure of accreting neutron stars. We find model-independent evidence for the onset of the $N=34$ subshell for Sc and the likely absence of $N=40$ magicity for $\mathrm{Cr}$, each of which result in a larger mass difference for transitioning from odd- $Z$ to odd- $N$ in EC. This is ultimately connected to the strength of the interaction between a nuclear core and an unpaired proton as opposed to an unpaired neutron [16, 59], and leads to increasing the phase-space available for the weak transitions involved in urca cooling, which in turn results in a cooler neutron star crust. Our measurements leave $\operatorname{ME}\left({ }^{63} \mathrm{~V}\right)$ as the final nuclear mass important for urca cooling in the accreted neutron star crust that relies on theoretical mass estimates.

This work was funded by the U.S. Department of Energy Office of Science through Grants No. DEFG02-88ER40387, de-sc0019042, de-sc0020451, and desc0020406 the U.S. National Science Foundation through Grants No. PHY-0822648, PHY-1102511, PHY-1811855, PHY-1913554, and PHY-1430152 (Joint Institute for Nuclear Astrophysics - Center for the Evolution of the Elements); and the DFG under Contracts No. GE2183/1-1 and No. GE2183/2-1.

* meisel@ohio.edu

[1] H. Schatz and W.-J. Ong, Astrophys. J. 844, 139 (2017).

[2] M. R. Mumpower, R. Surman, D. L. Fang, M. Beard, P. Möller, T. Kawano, and A. Aprahamian, Phys. Rev. C 92, 035807 (2015).

[3] D. Lunney, J. M. Pearson, and C. Thibault, Rev. Mod. Phys. 75, 1021 (2003)

[4] J. Duflo and A. Zuker, Phys. Rev. C 52, 23(R) (1995).

[5] S. Goriely, N. Chamel, and J. M. Pearson, Phys. Rev. C 82, 035804 (2010).

[6] P. Möller, W. D. Myers, H. Sagawa, and S. Yoshida, Phys. Rev. Lett. 108, 052501 (2012).

[7] N. Wang and M. Liu, J. Phys. Conf. Ser. 420, 012057 (2013)

[8] C. Thibault et al., Phys. Rev. C 12, 644 (1975)
[9] F. Wienholtz et al., Nature 498, 346 (2013).

[10] Z. Meisel et al., Phys. Rev. Lett. 114, 022501 (2015).

[11] M. Rosenbusch et al., Phys. Rev. Lett. 114, 202501 (2015)

[12] E. Leistenschneider et al., Phys. Rev. Lett. 120, 062503 (2018).

[13] M. Mougeot et al., Phys. Rev. Lett. 120, 232501 (2018)

[14] S. Michimasa et al., Phys. Rev. Lett. 121, 022506 (2018)

[15] X. Xu et al., Phys. Rev. C 99, 064303 (2019)

[16] S. Naimi et al., Phys. Rev. C 86, 014325 (2012).

[17] R. V. F. Janssens et al., Phys. Lett. B 546, 55 (2002).

[18] S. N. Liddick et al., Phys. Rev. Lett. 92, 072502 (2004)

[19] D. C. Dinca et al., Phys. Rev. C 71, 041302(R) (2005)

[20] H. L. Crawford et al., Phys. Rev. C 82, 014311 (2010)

[21] M. Hannawald et al., Phys. Rev. Lett. 82, 1391 (1999)

[22] O. Sorlin et al., Phys. Rev. Lett. 88, 092501 (2002)

[23] A. Gade et al., Phys. Rev. C 81, 051304(R) (2010)

[24] T. Baugher et al., Phys. Rev. C 86, 011305(R) (2012)

[25] Z. Meisel, A. Deibel, L. Keek, P. Shternin, and J. Elfritz, J. Phys. G 45, 093001 (2018).

[26] S. Gupta, E. F. Brown, H. Schatz, P. Möller, and K.-L. Kratz, Astrophys. J. 662, 1188 (2007).

[27] H. Schatz et al., Nature 505, 62 (2014).

[28] A. Estradé et al., Phys. Rev. Lett. 107, 172503 (2011)

[29] Z. Meisel et al., Phys. Rev. Lett. 115, 162501 (2015).

[30] Z. Meisel et al., Phys. Rev. C 93, 035805 (2016).

[31] M. Matoš et al., Nucl. Instrum. Methods Phys. Res., Sect. A 696, 171 (2012)

[32] Z. Meisel and S. George, Int. J. Mass Spectrom. 349-350, 145 (2013)

[33] D. J. Morrissey, B. M. Sherrill, M. Steiner, A. Stolz, and I. Wiedenhoever, Nucl. Instrum. Methods Phys. Res., Sect. B 204, 90 (2003).

[34] D. Bazin, J. Caggiano, B. Sherrill, J. Yurkon, and A. Zeller, Nucl. Instrum. Methods Phys. Res., Sect. B 204, 629 (2003)

[35] J. Yurkon et al., Nucl. Instrum. Methods Phys. Res., Sect. A 422, 291 (1999).

[36] D. Shapira, T. Lewis, and L. Hulett, Nucl. Instrum. Methods Phys. Res., Sect. A 454, 409 (2000)

[37] A. M. Rogers et al., Nucl. Instrum. Methods Phys. Res., Sect. A 795, 325 (2015)

[38] W. H. Press, S. A. Teukolsky, W. T. Vetterling, and B. P. Flannery, Numerical Recipes in C: The Art of Scientific Computing, 2nd ed. (Cambridge University Press, 1992).

[39] M. Wang, G. Audi, F. G. Kondev, W. J. Huang, S. Naimi, and X. Xu, Chin. Phys. C 41, 030003 (2017).

[40] C. Izzo et al., Phys. Rev. C 97, 014309 (2018).

[41] M. P. Reiter et al., Phys. Rev. C 98, 024310 (2018)

[42] M. Sawicka et al., Euro. Phys. J. A 16, 51 (2003).

[43] S. Lunardi et al., Phys. Rev. C 76, 034303 (2007)

[44] S. M. Lenzi, F. Nowacki, A. Poves, and K. Sieja, Phys. Rev. C 82, 054301 (2010).

[45] D. Steppenbeck et al., Phys. Rev. C 96, 064310 (2017)

[46] B. A. Brown, Phys. Rev. Lett. 111, 162502 (2013).

[47] M. Honma, T. Otsuka, B. A. Brown, and T. Mizusaki, Euro. Phys. J. A Suppl. 25, 499 (2005).

[48] O. Sorlin et al., Euro. Phys. J. A 16, 55 (2003).

[49] H. L. Crawford et al., Phys. Rev. Lett. 110, 242701 (2013)

[50] S. Tsuruta and A. Cameron, Astrophys. Space Sci. 7, 374 (1970).

[51] A. Deibel, Z. Meisel, H. Schatz, E. F. Brown, and A. Cumming, Astrophys. J. 831, 13 (2016). 
[52] Z. Meisel and A. Deibel, Astrophys. J. 837, 73 (2017)

[53] S. S. Gupta, T. Kawano, and P. Möller, Phys. Rev. Lett. 101, 231101 (2008)

[54] S. A. Moszkowski, Phys. Rev. 82, 35 (1951)

[55] B. Singh, J. L. Rodriguez, S. S. M. Wong, and J. K. Tuli, Nuc. Data Sheets 84, 487 (1998).
56] Z. Meisel, EPJ Web Conf. 178, 04004 (2018)

[57] W. J. Ong, Private communication (2019).

[58] Z. Meisel, G. Merz, and S. Medvid, Astrophys. J. 872, $84(2019)$

[59] N. Zeldes, M. Gronau, and A. Lev, Nucl. Phys. 63, 1 (1965) 